\title{
Identificación de las principales fuentes de agua que aportan a la generación de escorrentía en zonas Andinas de páramo húmedo: mediante el uso de los isótopos estables deuterio $\left(\delta^{2} \mathrm{H}\right)$ y oxígeno-18 $\left(\delta^{18} \mathrm{O}\right)$
}

\author{
Giovanny Mosquera, Patricio Lazo, Irene Cárdenas, Patricio Crespo \\ Grupo de Ciencias de la Tierra y del Ambiente, DIUC, Universidad de Cuenca, Campus Quinta \\ Balzay, Av. Víctor Manuel Albornoz, Cuenca, Ecuador
}

Autor para correspondencia: patricio.crespo@ucuenca.edu.ec

Fecha de recepción: 12 de octubre 2012 - Fecha de aceptación: 17 de enero 2013

\section{RESUMEN}

El análisis hidrológico de cuencas de páramo Andino actualmente se encuentra estancado debido a la limitada existencia de estudios sobre los procesos de generación de escorrentía. Para mejorar esta situación y proveer una línea base para futuros estudios hidrológicos, se realizó un análisis del fraccionamiento isotópico de deuterio $\left({ }^{2} \mathrm{H}\right)$ y oxígeno-18 $\left({ }^{18} \mathrm{O}\right)$ en muestras de agua recolectadas quincenalmente entre mayo del 2011 y marzo del 2012 en la cuenca de páramo húmedo del Río Zhurucay $\left(7,97 \mathrm{~km}^{2}\right)$, ubicada al sur del Ecuador, entre los 3200 y $3800 \mathrm{~m}$ s.n.m. Los puntos de monitoreo incluyen estaciones de lluvia, caudal, agua en el suelo, y manantiales. Los resultados revelan que el agua de pre-evento es la principal fuente de generación de escorrentía durante épocas lluviosas, siendo los Histosoles las principales fuentes de aporte a la descarga. Los Andosoles tienen como función principal la de regular el caudal y recargar los Histosoles durante condiciones normales principalmente debido a su posición en el paisaje (sobre los Histosoles). Por otra parte, durante condiciones secas los resultados sugieren que los Histosoles son la principal fuente de regulación de caudal. De la misma manera, no existen evidencias de aporte significativo de aguas subterráneas a la generación de caudal en la zona.

Palabras clave: Páramo Andino, procesos hidrológicos, isótopos estables, deuterio, oxígeno-18, Ecuador.

\begin{abstract}
The hydrologic analysis of Andean páramo catchments is currently hindered by the limited existence of studies regarding the streamflow generation processes. To improve this situation and to provide a baseline for future hydrologic studies, an isotope fractionation analysis of deuterium $\left({ }^{2} \mathrm{H}\right)$ and oxygen$18\left({ }^{18} \mathrm{O}\right)$ was conducted on water samples collected biweekly between May 2011 and March 2012, within the Zhurucay river páramo micro-catchment $\left(7,97 \mathrm{~km}^{2}\right)$, located in the Ecuadorian austral region, between 3200 and $3800 \mathrm{~m}$ a.s.l. The monitoring points encompassed rainfall and runoff stations, and soil and spring water sampling points. Results reveal that pre-event water is the main source for streamflow generation during storm conditions, where the Histosols are the main contribution to the discharge. Andosols main function is to regulate runoff and recharge Histosols during normal conditions. The latter are recharged by virtue of their low-lying position in the landscape. During less humid conditions, results suggest that the Histosols are the main sources of discharge regulation. Similarly, there is no evidence of significant groundwater contribution to discharge.
\end{abstract}

Keywords: Andean páramo, hydrologic processes, stable isotopes, deuterium, oxygen-18, Ecuador. 


\section{INTRODUCCIÓN}

En las regiones montañosas de América del Sur, la comprensión del funcionamiento hidrológico de los ecosistemas de páramo Andino es de vital importancia. Esto, no sólo debido a la creciente importancia de éstos ecosistemas como las principales fuentes de generación y regulación de agua, sino también porque dicha agua normalmente es de alta calidad. El agua proveniente de las zonas de páramo es utilizada entre otros fines para consumo humano, industrial, riego, agricultura y ganadería, generación energética, minería y recreación, actividades que permiten mantener el desarrollo de comunidades y sociedades que habitan dichas regiones y sus alrededores, tales como: Bogotá, Quito y Cuenca (De Bièvre y col., 2006; Buytaert y col., 2006b). Adicionalmente, el funcionamiento hidrológico permite mantener las características bio-ecológicas únicas que presentan este tipo de ecosistemas que actualmente se encuentran en riesgo principalmente debido a procesos antropogénicos. En tal sentido, mientras un gran número de estudios han sido realizados alrededor del mundo en una gran diversidad de ecosistemas con el fin de determinar sus funciones hídricas, en el caso de los ecosistemas de páramo húmedo alto-andino, existe una escasez de conocimiento que permita mejorar el entendimiento de dichos procesos. Además, en conjunto con el rápido aumento de la presión antropogénica sobre la dinámica de las cuencas de páramo, se encuentra la importancia de la conservación de las mismas de forma que se pueda mantener un caudal que permita el desarrollo de un funcionamiento normal/natural de los ecosistemas bióticos y abióticos, y de las especies de flora y fauna que de ellos dependen (caudal ecológico). Mismos que, a través del control de la calidad y cantidad de agua, el control de los procesos erosivos y la determinación de las fuentes de agua que aportan a la generación de escorrentía, son la base para un manejo sustentable de los recursos hídricos (Buytaert y col., 2006a; Mena y Hofstede, 2006).

Sin embargo, y a pesar de la gran variedad de servicios ambientales que los ecosistemas de páramo proveen a la sociedad, hasta el momento, existen pocos estudios sobre los procesos hidrológicos que gobiernan a los mismos (Díaz-Granados y col., 2005), y aunque en los últimos años, se han realizado esfuerzos significativos para mejorar el conocimiento sobre éstos, aún hace falta mucha investigación (Crespo y col., 2011a; Buytaert y col., 2006a). Razón por la cual, hasta el momento, existe discrepancia sobre el comportamiento hidrológico del páramo a escala de cuenca (Buytaert y col., 2004a), lo que genera un contraste con la importancia socioeconómica que tiene el mismo como fuente primaria de producción de agua.

$\mathrm{Al}$ respecto, a través del uso de información hidrométrica en cuencas pequeñas, hasta ahora en Ecuador no ha sido posible determinar estimaciones globales de los principales procesos que intervienen en la generación de caudales (Buytaert y col., 2004b, Crespo y col., 2011b), aunque de todas formas, los hidrosistemas de páramo son considerados reguladores naturales de escorrentía. Lo cual, junto a una alta capacidad de producción de agua, ha desembocado en que dicha capacidad de regulación de agua sea considerada casi legendaria entre agricultores y científicos (Hofstede, 1995; Medina y Mena, 2001). De hecho, muchos ríos de páramo proveen un flujo base sostenido a través del año (Buytaert y col., 2004b). En este sentido, al momento, se atribuye dicha capacidad de regulación de escorrentía básicamente a las condiciones climáticas, topográficas y de los suelos.

En consecuencia, con el fin de aclarar tales discrepancias, se ha vuelto cada vez más imperiosa e importante la necesidad de implementar el uso de nuevas técnicas y/o métodos que permitan profundizar el conocimiento del funcionamiento de los sistemas hidrológicos en zonas de páramo Andino. En tal sentido, como mencionan Kendall y McDonnell (1998), y Yurtsever (2000), de todos los métodos utilizados actualmente para modelar procesos hidrológicos a escala de pequeñas cuencas, el uso de trazadores isotópicos se ha constituido en una herramienta ideal que permite mejorar el entendimiento de dichos procesos a través de la determinación de la edad, origen y ruta del movimiento del agua. Esto, debido primordialmente a que son fáciles de medir, y que los periodos de monitoreo requeridos para establecer conclusiones determinantes sobre los mecanismos de generación de escorrentía, son relativamente cortos (Wilcox, 2011). En este aspecto, aunque hasta hace poco tiempo el uso de este tipo de técnicas se encontraba limitado debido principalmente a la falta de recursos económicos, en los últimos años diferentes grupos de investigación han empezado a luchar contra las difíciles condiciones del terreno y el escaso financiamiento, con el fin de mejorar el conocimiento sobre la hidrología Andina. Conocimiento desde el cual se pretende partir en la 
búsqueda del desarrollo de nuevas políticas de conservación y recuperación de dichos ecosistemas (Crespo y col., 2011a).

De esta forma, a través del uso combinado de información hidrométrica y de los trazadores isotópicos ambientales, deuterio $\left({ }^{2} \mathrm{H}\right)$ y oxígeno-18 $\left({ }^{18} \mathrm{O}\right)$, este estudio busca profundizar el conocimiento de los procesos hidrológicos que se llevan a cabo a escala de cuenca en la región Andina, y adicionalmente establecer una línea base que permita desarrollar estudios isotópicos futuros a escala de cuenca. Consecuentemente, los objetivos de la investigación fueron: i) caracterizar los isótopos de $\delta^{2} \mathrm{H}$ y $\delta^{18} \mathrm{O}$ en la precipitación y su comportamiento en el tiempo y a diferentes altitudes, ii) analizar el fraccionamiento de $\delta^{2} \mathrm{H}$ y $\delta^{18} \mathrm{O}$ en el caudal, suelo y el agua de manantiales (spring water), y iii) identificar las principales fuentes de generación de escorrentía.

\section{MATERIALES Y MÉTODOS}

\subsection{Descripción área de estudio}

La zona de estudio (microcuenca del Río Zhurucay), se encuentra ubicada al sur del Ecuador, aproximadamente a $85 \mathrm{~km}$ al suroeste de la ciudad de Cuenca, en los páramos de Quimsacocha. La cuenca del Río Zhurucay tiene un área de drenaje de $7,97 \mathrm{~km}^{2}$ y un rango altitudinal entre los $3400 \mathrm{y}$ 3900 m s.n.m. El Río Zhurucay es afluente del Río Jubones que desemboca al océano Pacífico. El clima de la cuenca de estudio está influenciado por el régimen del Pacífico que llega del oeste y las masas de aire continentales del lado Amazónico que provienen del Atlántico (Vuille y col., 2000). Como resultado de la influencia de estos dos regímenes la precipitación anual resultante es ligeramente bimodal (con una estación más seca entre Agosto a Septiembre y otra menos seca de Diciembre a Febrero) ya que la distribución anual de la precipitación es relativamente uniforme (Buytaert y col., 2005). La lluvia media anual está entre 900 y $1600 \mathrm{~mm}$ entre el periodo 1964-2009 (a partir de datos de las estaciones del INAMHI, Instituto Nacional de Meteorología e Hidrología del Ecuador). La lluvia es frecuente durante el año y está caracterizada por intensidades bajas (alrededor del $90 \%$ por debajo de los $10 \mathrm{~mm} \mathrm{~h}^{-1}$ ) (Buytaert y col., 2006a). La temperatura del aire decrece con la altitud con una gradiente térmica promedio de 0,5 a $0,7^{\circ} \mathrm{C}$ por cada $100 \mathrm{~m}$ (Van der Hammen y Hooghiemstra, 2000; Castaño, 2002; Baculima y col., 1999), con una temperatura media de $7^{\circ} \mathrm{C}$ a 3500 m s.n.m. (Crespo y col., 2011a).

En relación a la geología de la cuenca de estudio, en la zona existen tres formaciones: La formación Turi (brechas tobáceas primarias de composición andesítica) perteneciente al Mioceno Tardío, la formación Quimsacocha (lavas y tobas) de edad incierta aunque probablemente podrían ser del Mioceno Tardío o más joven; y la formación Santa Isabel (lavas y brechas tobáceas andesíticas) atribuida al Mioceno Temprano. La litología que se puede identificar en la cuenca alta del Zhurucay son los flujos de lava de la formación Quimsacocha que sobre-yacen a los volcano-sedimentos de la formación Turi, mientras que en la cuenca media y baja se encuentran las formaciones Turi y Santa Isabel. Depósitos cuaternarios se ubican generalmente en las zonas de pendiente fuerte y escarpes. La cobertura vegetal de la cuenca de estudio está dominada principalmente por el pajonal, almohadillas y bosque de Polylepis sp. Los usos de la tierra están limitados al pastoreo extensivo de baja densidad animal y quemas en la parte baja de la cuenca. Los suelos más comunes en el área de estudio son los Andosoles e Histosoles (Crespo y col., 2011a) según la clasificación de la FAO (FAO/ISRIC/ISSS, 1998).

\subsection{Recolección de información hidrométrica}

Para la medición de los caudales dentro de la cuenca del Río Zhurucay se usó un sistema de monitoreo anidado "nested approach", compuesto por diez microcuencas, donde las microcuencas M1 a M10 son tributarias y la M9 corresponde a la salida de la cuenca de estudio (Figura 1 y Tabla 1). 


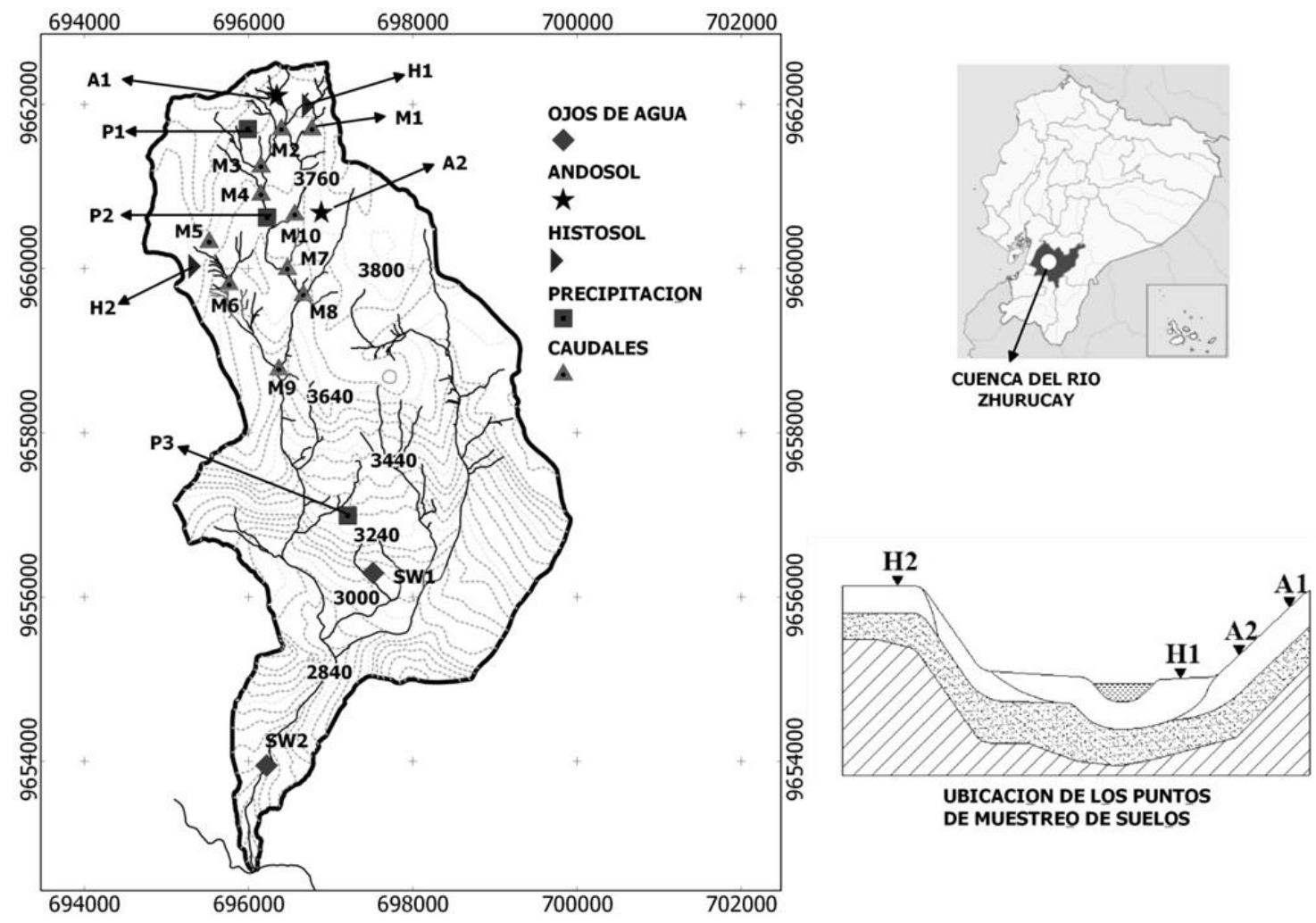

Figura 1. Ubicación del área de estudio y sitios de monitoreo en la cuenca del Río Zhurucay para Caudal (M), Precipitación (P), Andosoles (A), Histosoles (H) y Agua de Manantial (SW). (En la parte inferior derecha, se puede observar un corte transversal de la ubicación de las estaciones de monitoreo de suelos sobre el paisaje en la cuenca de estudio).

Tabla 1. Características fisiográficas e hidrométricas de las microcuencas monitoreadas en la cuenca del Río Zhurucay.

\begin{tabular}{|c|c|c|c|c|c|c|c|}
\hline \multirow[t]{2}{*}{$\begin{array}{l}\text { Micro- } \\
\text { cuenca }\end{array}$} & \multirow{2}{*}{$\begin{array}{l}\text { Área } \\
\left(\mathrm{km}^{2}\right)\end{array}$} & \multicolumn{2}{|c|}{$\begin{array}{l}\text { Rango altitudinal } \\
\text { (ms.n.m.) }\end{array}$} & \multirow{2}{*}{$\begin{array}{c}\text { Pendiente } \\
\text { media } \\
(\%)\end{array}$} & \multirow{2}{*}{$\begin{array}{l}\text { Precipitación } \\
(\mathrm{mm} \text { año }\end{array}$} & \multirow{2}{*}{$\begin{array}{c}\text { Caudal } \\
\left(\mathrm{mm} \mathrm{año}^{-1}\right)\end{array}$} & \multirow[t]{2}{*}{$\begin{array}{l}\text { Coeficiente } \\
\text { de escorrentía }\end{array}$} \\
\hline & & Min & Max & & & & \\
\hline M1 & 0,20 & 3777 & 3900 & 16 & 1278 & 924 & 0,72 \\
\hline M2 & 0,38 & 3770 & 3900 & 19 & 1278 & 818 & 0,64 \\
\hline M3 & 0,38 & 3723 & 3850 & 15 & 1264 & 565 & 0,45 \\
\hline M4 & 0,65 & 3715 & 3850 & 14 & 1269 & 508 & 0,40 \\
\hline M5 & 0,89 & 3771 & 3830 & 3 & 1144 & 645 & 0,56 \\
\hline M6 & 1,66 & 3645 & 3830 & 8 & 1160 & 511 & 0,44 \\
\hline M7 & 3,28 & 3676 & 3900 & 9 & 1240 & 678 & 0,55 \\
\hline M8 & 0,87 & 3664 & 3830 & 11 & - & - & - \\
\hline M9 & 7,57 & 3505 & 3900 & 11 & 1264 & 1082 & 0,86 \\
\hline M10 & 1,34 & 3680 & 3900 & 18 & - & - & - \\
\hline
\end{tabular}

Para la estimación del caudal se usaron vertederos triangulares compuestos de pared delgada " $90^{\circ}$ V-notch weirs" en las microcuencas tributarias, mientras que a la salida de la cuenca de estudio (M9) se usó un vertedero rectangular de pared delgada. Cada vertedero estuvo provisto por un sensor de nivel de agua tipo transductor de presión marca Schlumberger con una precisión de +/-5 mm. Estos 
niveles fueron medidos en intervalos de cinco minutos y transformados a caudal mediante el uso de las relaciones de Kindsvater-Shen. Estas ecuaciones fueron validadas y corregidas usando mediciones de caudal rutinarias, para lo cual se usó el método de aforos de dilución de sal. Para la recolección de los datos de precipitación se utilizaron pluviógrafos tipo balancín de 0,2 y $0,254 \mathrm{~mm}$ de capacidad, de marcas ONSET y DAVIS respectivamente, ubicados en diferentes puntos estratégicos de la cuenca de estudio. Debido a que la diferencia en la cantidad de precipitación en el periodo de minitoreo entre las diferentes estaciones es pequeña (Tabla 1), la precipitación media en las diferentes microcuencas fue calculada utilizando el método de Polígonos de Thiessen.

Debido a las condiciones meteorológicas hubo algunos problemas en el funcionamiento de los equipos, produciéndose vacíos en la información que fueron rellenados mediante el uso de correlaciones entre equipos cercanos en el caso de la precipitación, mientras que, en el caso de los niveles/caudales, los vacíos en algunas microcuencas (M8 y M10) no pudieron ser rellenados debido a que no se contaba con información suficiente.

\subsection{Recolección de información isotópica}

La información isotópica de $\delta^{18} \mathrm{O}$ y $\delta^{2} \mathrm{H}$ fue recolectada entre mayo de 2011 y marzo de 2012 de manera quincenal en la cuenca de estudio. Para la recolección de información isotópica en el agua de los ríos se utilizó el mismo sistema de monitoreo anidado "nested approach" utilizado para la recolección de información hidrométrica. Adicionalmente, se recolectaron muestras de agua en la precipitación, en los diferentes horizontes del suelo y en el agua de manantiales (spring water). En la Figura 1, se encuentran representados los puntos de muestreo tanto para lluvia (P), escorrentía (M), agua de manantial (spring water, SW), así como para cada tipo de suelo, Histosoles (H) y Andosoles (A). Cabe resaltar que durante algunas jornadas de muestreo durante épocas secas no se pudieron recolectar muestras de precipitación debido a que no se encontró agua en los recolectores.

Para el monitoreo isotópico de la precipitación se seleccionaron tres puntos ubicados en la parte alta (P1), media (P2) y baja (P3) de la cuenca a 3779, 3700 y 3297 m s.n.m respectivamente, como se muestra en la Figura 1. Las muestras de agua en los ríos fueron recolectadas en diez puntos (M1 M10) ubicados en las partes alta y media de la cuenca cerca de cada uno de los vertederos existentes para la medición del caudal, así como también a la salida de la misma (M9, confluencia de las microcuencas). Para el muestreo de los suelos se escogieron cuatro puntos a lo largo de la cuenca de estudio, dos en zona de humedal (Histosoles) y dos en pajonal (Andosoles). Los sitios de muestreo de suelos fueron seleccionados estratégicamente para representar sus diferentes condiciones de drenaje. En la Figura 1 se puede observar un corte transversal de la cuenca de estudio, donde se presenta la ubicación de las estaciones de monitoreo de suelos sobre el paisaje en la misma. De forma que, el primer punto de monitoreo en humedales (Histosol 1) se encuentra situado en la zona baja de una ladera, mientras que el segundo punto (Histosol 2) se encuentra ubicado en una zona plana a la salida de un gran humedal denominado "lagunas ciegas" (zona de humedales confinados). En el caso de los pajonales, ambos puntos se encuentran ubicados sobre laderas; sin embargo, difieren en la posición de cada uno sobre las mismas. El primero (Andosol 1) se sitúa en la parte media de la ladera, mientras que el segundo (Andosol 2) se ubica en la parte baja. En cada sitio de monitoreo de suelos se recolectaron muestras en los horizontes orgánico y mineral. En los Andosoles se colocaron dos platos de succión sobre el horizonte orgánico a 25 y $35 \mathrm{~cm}$ de profundidad, y uno en el horizonte mineral a $65 \mathrm{~cm}$ de profundidad. De igual forma, en los Histosoles se colocaron platos de succión sobre el horizonte orgánico a 25 y $45 \mathrm{~cm}$ de profundidad, y a $75 \mathrm{~cm}$ de profundidad en el horizonte mineral, esto debido a que en el caso de los Histosoles, el horizonte mineral se encontró ubicado a mayor profundidad. Finalmente, se recolectaron muestras de agua que fluye a través de las grietas en la roca, para lo cual se monitorearon dos manantiales (spring water), los cuales se encuentran ubicados a 3282 y 2656 m s.n.m., con la finalidad de verificar si existe influencia de aguas subterráneas en la generación de escorrentía en la cuenca.

Las muestras de agua fueron tomadas y almacenadas en botellas de vidrio ámbar de $2 \mathrm{ml}$, las cuales adicionalmente fueron cubiertas con parafilm y almacenadas en lugares oscuros con el fin de evitar el fraccionamiento por evaporación hasta su posterior análisis en el laboratorio, como recomienda la AIEA (Agencia Internacional de Energía Atómica). La toma de muestras en caudales y 
los manantiales se la realizó directamente de los cauces, en zonas donde el agua se encontraba circulando regularmente (evitando tomar las mismas en zonas de estancamiento). Las muestras de agua en los suelos fueron extraídas usando platos de succión (suction plates) con una succión de $1 \mathrm{~m}$, y posteriormente recolectadas y almacenadas en botellas de vidrio. Luego de cada muestreo se procedió a vaciar y limpiar las botellas recolectoras para evitar su contaminación. Las muestras de precipitación fueron tomadas mediante el uso de recolectores de lluvia de polipropileno cubiertos con papel de aluminio para disminuir la evaporación del agua. Adicionalmente, posterior al recolectado de muestras de precipitación, el recolector de agua lluvia fue secado, limpiado y se colocó aceite de vaselina antes de volver a instalarlo, para evitar un posible fraccionamiento por la evaporación del agua recolectada entre cada jornada de monitoreo. En el caso de las muestras recolectadas en los suelos y la precipitación, una vez tomadas las mismas, se procedió a cuantificar el volumen de agua almacenada en el recolector.

\subsection{Análisis de laboratorio}

El análisis de muestras se realizó mediante el uso de un cavity ring-down spectrometer utilizando el Analizador Isotópico de Agua y Vapor de Agua Picarro L1102-i, con una precisión de 0,5\%o para deuterium y de $0,1 \%$ para oxígeno-18. Se usaron tres estándares isotópicos de diferente valor para verificar la calidad de los análisis. En este sentido, por un lado se observó la linealidad de los resultados (valores de $\mathrm{R}^{2}>0,99$ ) y por otro la precisión de los análisis de las distintas repeticiones. Para mantener una alta calidad de los datos, también se realizaron pruebas periódicas de calibración de la máquina, denominadas "Drift Test". Los resultados de las muestras isotópicas se presentan mediante valores de abundancia isotópica $(\delta)$, los cuales se expresan en unidades por mil (\%o) con respecto al estándar internacional VSMOW (Vienna Standard Mean Ocean Water) para Oxígeno-18 y Deuterio, el cual establece un valor de referencia de $0 \%$ para la abundancia isotópica del agua oceánica.

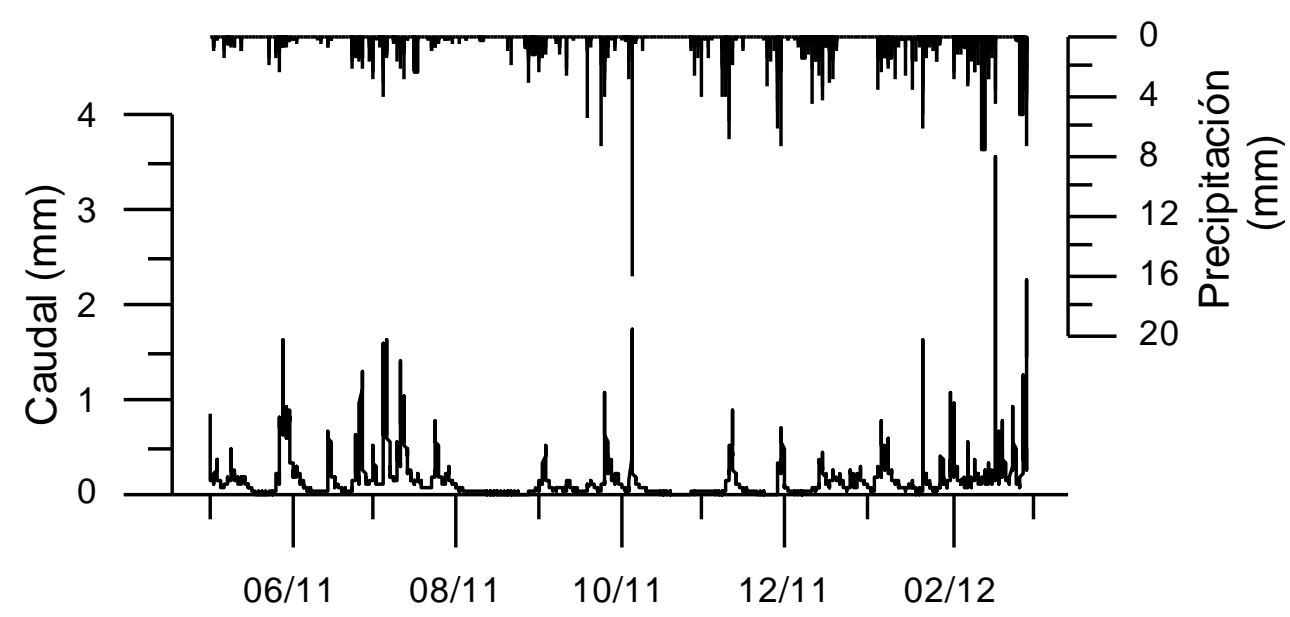

Figura 2. Relación horaria entre la precipitación-caudal en la microcuenca M5 de la cuenca del Río Zhurucay.

\section{RESULTADOS Y DISCUSIÓN}

\subsection{Relación lluvia - caudal}

En relación a la información hidrométrica, en la cuenca de estudio se observa que la precipitación acumulada en las diferentes microcuencas monitoreadas exhiben una variación entre 1144 y $1278 \mathrm{~mm}$ año ${ }^{-1}$ (Tabla 1). Dichos valores evidencian que la distribución espacial de la precipitación en la cuenca es prácticamente homogénea debido a que no existen diferencias significativas en la cantidad de lluvia precipitada a lo largo de la misma. Adicionalmente, como se puede observar en la Figura 2, la 
intensidad de la precipitación es baja durante todo el periodo de monitoreo, exhibiendo intensidades menores a $10 \mathrm{~mm} \mathrm{~h}^{-1}$, lo que concuerda con los estudios previos de Crespo y col. (2011b) y Buytaert y col. (2005) realizados en cuencas ubicadas en zonas del austro Ecuatoriano, en donde se encontraron tendencias similares.

Tabla 2. Valores máximos, mínimos, promedios y desviaciones estándar de las señales isotópicas de $\delta^{18} \mathrm{O}$ en la cuenca del Río Zhurucay para caudal (M), precipitación, suelos (Andosoles e Histosoles a diferentes profundidades) y agua de manantial (SW). $(-25,25 \mathrm{~cm}$ de profundidad; $-35,35 \mathrm{~cm}$ de profundidad; $-45,45 \mathrm{~cm}$ de profundidad; $-65,65 \mathrm{~cm}$ de profundidad; $-75,75 \mathrm{~cm}$ de profundidad).

\begin{tabular}{|c|c|c|c|c|c|}
\hline \multirow{2}{*}{$\begin{array}{l}\text { Estación de } \\
\text { muestreo }\end{array}$} & \multirow{2}{*}{$\begin{array}{l}\text { Elevación } \\
\text { (m s.n.m) }\end{array}$} & \multicolumn{4}{|c|}{$\delta^{18} \mathrm{O}(\%)$} \\
\hline & & Max & Min & Promedio & $\begin{array}{c}\text { Desviación } \\
\text { estándar }\end{array}$ \\
\hline M1 & 3777 & $-9,8$ & $-16,2$ & $-12,0$ & 1,5 \\
\hline M2 & 3770 & $-9,6$ & $-15,5$ & $-11,8$ & 1,3 \\
\hline M3 & 3723 & $-10,1$ & $-15,6$ & $-11,6$ & 1,4 \\
\hline M4 & 3715 & $-9,9$ & $-14,0$ & $-11,7$ & 1,3 \\
\hline M5 & 3771 & $-7,3$ & $-15,0$ & $-10,5$ & 2,3 \\
\hline M6 & 3645 & $-8,8$ & $-14,9$ & $-10,9$ & 1,5 \\
\hline M7 & 3676 & $-9,7$ & $-14,9$ & $-11,5$ & 1,3 \\
\hline M8 & 3664 & $-9,8$ & $-14,0$ & $-11,7$ & 1,4 \\
\hline M9 & 3505 & $-9,1$ & $-13,5$ & $-11,2$ & 1,3 \\
\hline M10 & 3697 & $-10,2$ & $-15,7$ & $-11,9$ & 1,4 \\
\hline Prec. Alta & 3779 & $-3,7$ & $-18,8$ & $-10,4$ & 3,7 \\
\hline Prec. Media & 3700 & $-4,8$ & $-16,4$ & $-10,6$ & 2,9 \\
\hline Prec. Baja & 3297 & $-2,9$ & $-14,7$ & $-8,8$ & 3,0 \\
\hline Andosol 1-25* & & $-6,8$ & $-15,7$ & $-10,9$ & 3,1 \\
\hline Andosol 1-35* & 3770 & $-8,7$ & $-13,6$ & $-11,1$ & 1,6 \\
\hline Andosol 1-65* & & $-9,0$ & $-13,0$ & $-11,4$ & 1,1 \\
\hline Andosol 2-25* & & $-7,4$ & $-15,5$ & $-11,2$ & 2,8 \\
\hline Andosol 2-35* & 3703 & $-8,4$ & $-15,3$ & $-11,1$ & 2,2 \\
\hline Andosol 2-65* & & $-7,7$ & $-15,3$ & $-11,0$ & 2,3 \\
\hline Histosol 1-25* & & $-10,0$ & $-13,3$ & $-11,3$ & 0,6 \\
\hline Histosol 1-45* & 3794 & $-10,3$ & $-13,0$ & $-11,2$ & 0,5 \\
\hline Histosol 1-75* & & $-10,5$ & $-12,4$ & $-11,3$ & 0,4 \\
\hline Histosol 2-25* & & $-6,6$ & $-14,2$ & $-9,9$ & 2,5 \\
\hline Histosol 2-45* & 3777 & $-7,7$ & $-9,0$ & $-8,3$ & 0,5 \\
\hline Histosol 2-75* & & $-7,3$ & $-9,1$ & $-8,6$ & 0,6 \\
\hline SW1 & 3282 & $-10,1$ & $-10,6$ & $-10,5$ & 0,1 \\
\hline SW2 & 2656 & $-8,2$ & $-8,6$ & $-8,5$ & 0,1 \\
\hline
\end{tabular}

En cuanto al caudal, la variación en la generación de escorrentía a lo largo de la cuenca es mayor, presentándose valores de caudal acumulado que se encuentran en un rango entre 508 y $1082 \mathrm{~mm}$ año ${ }^{-1}$. Siendo los caudales de las microcuencas M1 y a la salida de la cuenca (M9) mayores con valores que varían entre 924 y $1082 \mathrm{~mm}_{\text {año }}{ }^{-1}$, lo cual se debe principalmente a una deficiente calibración de los vertederos de dichas cuencas. En las microcuencas M8 y M10 no fue posible estimar los caudales debido a la existencia de varios periodos con vacíos de información, por lo que se las ha excluido del 
análisis hidrométrico. Los coeficientes de escorrentía en la mayor parte de las microcuencas durante el periodo de monitoreo se encuentran entre 0,4 y 0,64 (Tabla 1), excepto para las microcuencas M1 y M9 por las causas previamente mencionadas. Dichas variaciones en el coeficiente de escorrentía podrían encontrarse relacionadas: i) a las diferencias en los tipos de cobertura vegetal de cada microcuenca, ii) a los diferentes usos de suelo en las mismas, y/o iii) a las características geológicas. Lamentablemente no es posible realizar un análisis más profundo debido a que no se contó con la información necesaria al momento de la publicación, por lo que dicho análisis será producto de posteriores estudios y publicaciones.

Finalmente, respecto a la relación lluvia-caudal, en la Figura 2 se puede observar que en la microcuenca 5 (M5) existe una rápida respuesta del caudal a ingresos de precipitación, tendencia general que se presenta en todas las microcuencas que forman parte del sistema hídrico de la cuenca del Río Zhurucay. Adicionalmente, dicha figura evidencia que la intensidad de la lluvia es baja como se mencionó anteriormente, siendo menor a los valores de conductividad hidráulica saturada del horizonte orgánico, los cuales varían entre 15 y $20 \mathrm{~mm} \mathrm{~h}^{-1}$ en los Andosoles ubicados entre los 40 y 60 $\mathrm{cm}$ de profundidad en la zona de estudio (Quichimbo y col., 2012). Resultados similares fueron encontrados por Buytaert y col. (2007); Crespo y col. (2010); PROMAS/IAMGOLD (2009). En conclusión, es muy improbable que exista flujo superficial por exceso de infiltración (flujo Hortoniano), siendo este posible únicamente durante cortos periodos de tiempo. Esto se ve reflejado en el evento sucedido a inicios de octubre del 2011 (Figura 2) donde se observa que el evento de lluvia de $16 \mathrm{~mm} \mathrm{~h}^{-1}$ no genera valores máximos de caudal, debido a las condiciones antecedentes de humedad del suelo. Resultados encontrados en otros estudios también excluyen el flujo Hortoniano en microcuencas de páramos de Ecuador y Chile (Buytaert y col., 2007; Blume y col., 2007).

\subsection{Señal isotópica}

A pesar de haber analizado la abundancia de $\delta^{2} \mathrm{H}$ y $\delta^{18} \mathrm{O}$, en el estudio se presentan únicamente los resultados obtenidos por medio de la abundancia de $\delta^{18} \mathrm{O}$, debido a que los resultados obtenidos mediante ambos isótopos muestran tendencias similares. En la Tabla 2 se presentan los valores máximos, mínimos, promedios y la desviación estándar de las abundancias isotópicas de $\delta^{18} \mathrm{O}$, junto con la elevación sobre el nivel del mar para todos los sitios monitoreados en la cuenca.

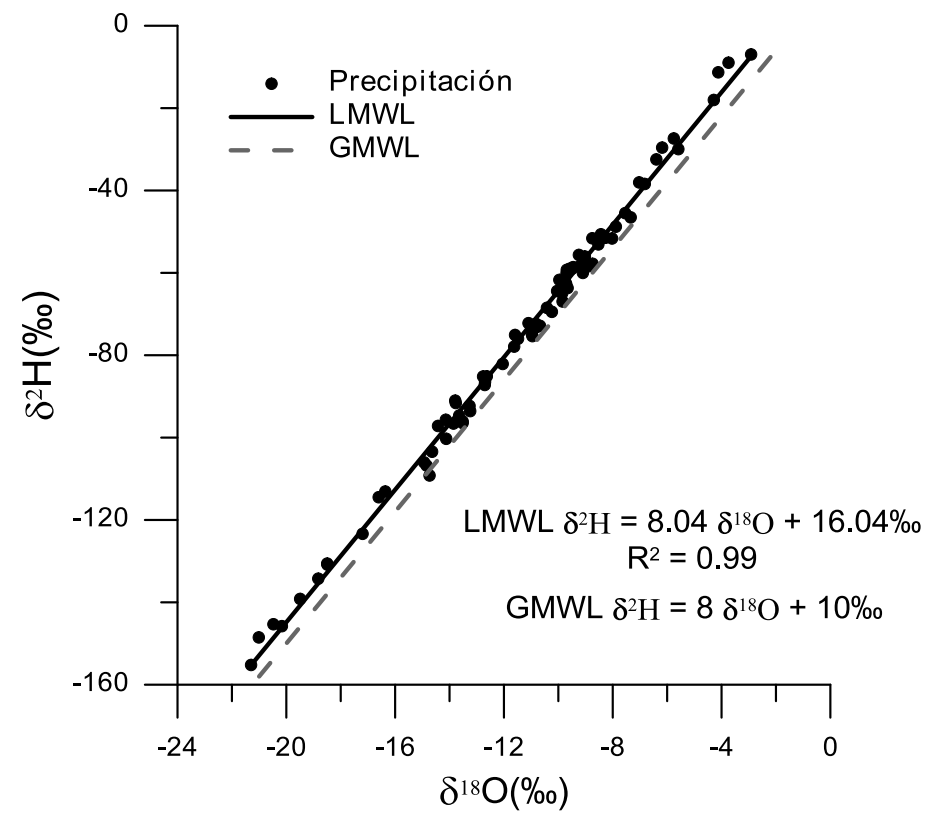

Figura 3. Relación entre los valores de $\delta^{18} \mathrm{O}$ de las muestras de precipitación obtenidas en la cuenca del Río Zhurucay (Línea de Agua Meteórica, LMWL). 


\subsection{Caracterización espacial y temporal de la abundancia isotópica de $\delta^{2} H$ y $\delta^{18} O$ en la precipitación}

Mediante el análisis de la relación entre las abundancias isotópicas de $\delta^{18} \mathrm{O}$ y $\delta^{2} \mathrm{H}$ de las muestras de precipitación recolectadas en la cuenca de estudio, se obtuvo la Línea de Agua Meteórica Local (LMWL, $\delta^{2} \mathrm{H}=8,04 \delta^{18} \mathrm{O}+16,04 \%$, Figura 3). La pendiente de la LMWL de 8,04, cercana a la de la Línea de Agua Meteórica Global (GMWL), cuyo valor es de 8, evidencia que el fraccionamiento generado por el proceso de evaporación es insignificante. Esto sugiere, que el fraccionamiento que se produce en la lluvia se realiza prácticamente en equilibrio en la zona de estudio. Este resultado se encuentra en línea con resultados obtenidos por otras investigaciones realizadas en el país, como la de Goller y col. (2005). Por otra parte, la LMWL exhibe un valor de exceso de deuterio de $16 \%$, enriquecido en relación al de la GMWL (10\%). Lo que posiblemente se debe a la existencia de lluvias localizadas enriquecidas al reevaporarse y/o a la presencia de lluvias provenientes de diversas fuentes de vapor de agua, como son el océano Pacífico y Atlántico. Similares resultados fueron obtenidos en un estudio realizado por Roche y col. (1999), basado en el análisis de la composición isotópica de la precipitación entre los Andes y la Amazonía bolivianos, donde menciona que los valores de exceso de deuterio determinados en estaciones de muestreo ubicadas a elevaciones mayores a $1000 \mathrm{~m}$ s.n.m., son mayores a $15 \%$.

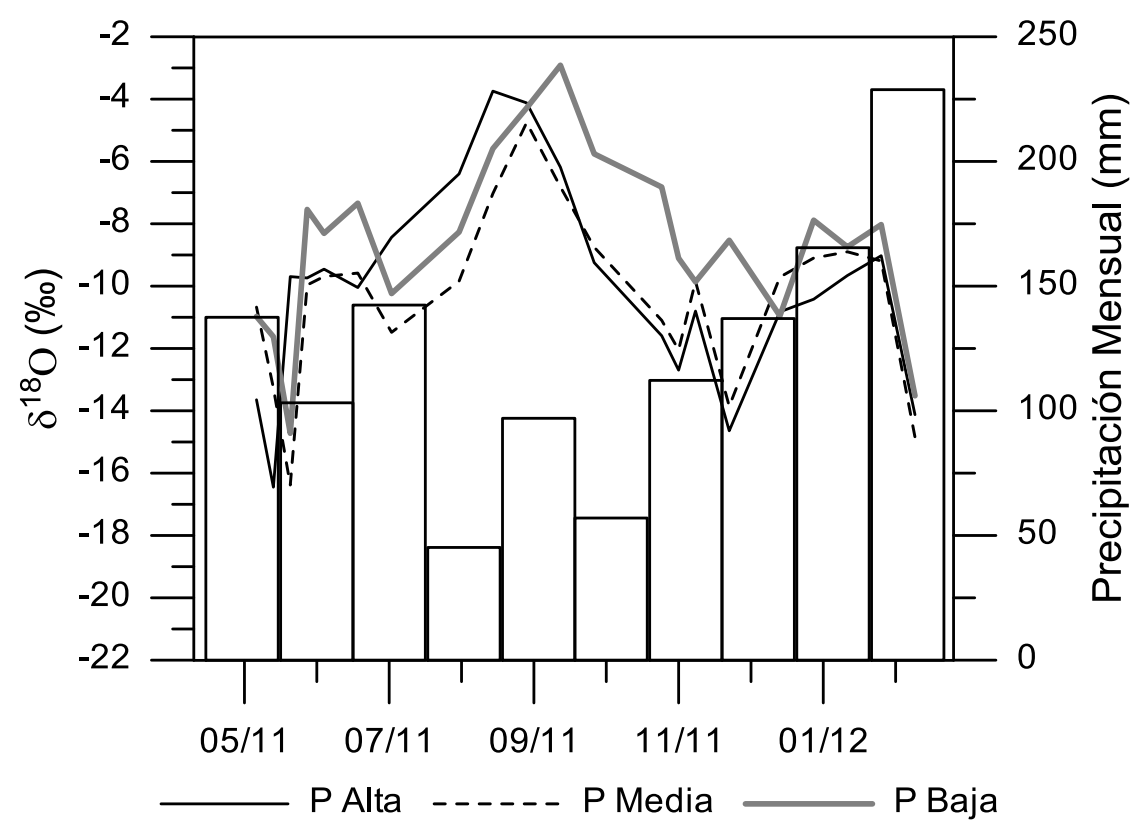

Figura 4. Señal isotópica de $\delta^{18} \mathrm{O}$ de la precipitación para las estaciones ubicadas en las partes alta (3779 m s.n.m.), media (3700 m s.n.m.) y baja (3297 m s.n.m.) de la cuenca en relación a la cantidad de precipitación mensual.

Con respecto a la abundancia de $\delta^{18} \mathrm{O}$ en la precipitación, los valores varían entre $-3,7 \%$ y $18,8 \%$ en la parte alta (3779 m s.n.m.), $-4,8 \%$ y $-16,4 \%$ en la parte media (3700 m s.n.m.) y $-2,9 \%$ y $-14,7 \%$ en la parte baja (3297 m s.n.m.) de la cuenca. Los valores promedios de $\delta^{18} \mathrm{O}$ para las partes alta, media y baja de la cuenca son de $-10,4 \%$, $-10,6 \%$ y $-8,8 \%$, respectivamente. Estos valores reflejan un claro empobrecimiento en abundancia isotópica de $\delta^{18} \mathrm{O}$ al incrementarse la elevación del punto de muestreo, lo que se denomina efecto altitudinal. Mediante el análisis de estos resultados, se ha determinado que para la cuenca monitoreada ubicada entre $\operatorname{los} 3200$ y 3800 m s.n.m., la abundancia de $\delta^{18} \mathrm{O}$ decrece a una razón aproximada de $0,5 \%$ por cada $100 \mathrm{~m}$ de incremento de altitud $\left(\mathrm{R}^{2}=0,95\right)$. Esto concuerda con el estudio de Rozanski y Araguas-Araguas (1995), sobre la variabilidad espacial y temporal de la precipitación en Sudamérica, basado en el análisis de la composición de isótopos estables en la lluvia, el cual determinó una tendencia similar entre la relación del $\delta^{18} \mathrm{O}$ de la precipitación y el incremento de altitud (aproximadamente $0,5 \% / 100 \mathrm{~m}$ ), en estaciones ubicadas sobre los 3000 m s.n.m. Al respecto, Vogel y col. (1975) determinaron la existencia del efecto de altitud en 
base al análisis de la abundancia de $\delta^{18} \mathrm{O}$ en aguas superficiales en los Andes de Argentina. En este estudio, se determinó un decremento aproximado de $0,2 \%$ en aguas superficiales, por cada $100 \mathrm{~m}$ de incremento de altitud en estaciones ubicadas a elevaciones entre 2000 y 4000 m s.n.m.

Sin embargo, durante algunos periodos, se puede observar una tendencia de empobrecimiento en la abundancia isotópica de $\delta^{18} \mathrm{O}$ en la precipitación en diferentes partes de la cuenca, relacionada al ingreso localizado de altas cantidades de lluvia con bajas abundancias isotópicas al sistema hidrológico (efecto de cantidad). Este efecto se puede observar claramente en las señales isotópicas de las muestras de precipitación recolectadas entre finales de junio y mediados de septiembre de 2011 (Figura 4), en las cuales los valores de $\delta^{18} \mathrm{O}$ en la parte media de la cuenca se encuentran empobrecidos en relación a los de la parte alta de la misma. Lo que aparentemente contrasta con el previamente mencionado efecto de altitud que se observa durante el resto del periodo de monitoreo en la cuenca, aunque, debido a la poca diferencia altitudinal entre las estaciones de monitoreo alta y media $(79 \mathrm{~m})$ en relación al efecto de cantidad, dichos resultados no son inesperados. Esto concuerda con el estudio sobre la composición isotópica estable de la precipitación de Roche y col. (1999), a lo largo de dos transectos entre el altiplano boliviano y la cuenca del Río Amazonas.

Así mismo, en la Figura 4 se observa una relación directa entre la señal isotópica de $\delta^{18} \mathrm{O}$ de la precipitación y la estacionalidad de la misma en el área de estudio. Durante épocas poco lluviosas (agosto a octubre de 2011) existe una tendencia de enriquecimiento en abundancia isotópica de la lluvia. En efecto, durante dicho periodo, se produjeron los valores más enriquecidos en abundancia isotópica de $\delta^{18} \mathrm{O}$ de la precipitación de $-3,7 \%$ o, $-4,8 \%$ y $-2,9 \%$ para las partes alta, media y baja de la cuenca, respectivamente. En contraste, durante periodos más húmedos (entre mayo y julio de 2011, y desde mediados de diciembre de 2011 hasta marzo de 2012) existe una tendencia al empobrecimiento en la abundancia de isótopos en la precipitación en respuesta al incremento en la intensidad de la lluvia. De hecho, durante dichos periodos, se llegaron a producir los valores más empobrecidos en la abundancia isotópica de $\delta^{18} \mathrm{O}$ de $-21,0 \%$ en la parte alta, $-21,3 \%$ en la parte media y $-17,2 \%$ en la parte baja de la cuenca. Resultados en línea con el estudio de García y col. (1998) obtenidos del análisis de registros a largo plazo de $\delta^{18} \mathrm{O}$ en la lluvia de la estación de Izobamba, Ecuador, ubicada a $3058 \mathrm{~m}$ s.n.m. que determinó la existencia de valores más empobrecidos en abundancia isotópica de $\delta^{18} \mathrm{O}$ en la lluvia durante temporadas de alta precipitación. Tendencias similares fueron encontradas en el estudio de Roche y col. (1999) sobre la composición isotópica de la lluvia en zonas andinas y amazónicas bolivianas.

\subsection{Relación entre las señales de $\delta^{18}$ O del agua de lluvia y el agua del suelo}

En la Tabla 2, se presentan los valores de las abundancias isotópicas de $\delta^{18} \mathrm{O}$ para el agua que circula a diferentes profundidades sobre cada tipo de suelo monitoreado. Los valores promedios de la señal isotópica de $\delta^{18} \mathrm{O}$ del Andosol 1 son de -10,9\% (Andosol 1-25), -11,1\%o (Andosol 1-35) y -11,4\%o (Andosol 1-65) para las muestras de agua recolectadas a 25,35 y $65 \mathrm{~cm}$ de profundidad, respectivamente. La abundancia isotópica de $\delta^{18} \mathrm{O}$ del Andosol 1 presenta una variación entre $-6,8 \%$ y $-15,7 \%$ a una profundidad de $25 \mathrm{~cm}$; mientras que en los niveles más profundos las señales isotópicas de $\delta^{18} \mathrm{O}$ se presentan más atenuadas a lo largo del periodo de monitoreo, con variaciones entre $-8,7 \%$ y $-13,6 \%$ en el Andosol 1-35, y $-9,0 \%$ y $-13,0 \%$ en el Andosol 1-65. Al analizar estos valores y la Figura 5a, se observa que en el Andosol 1, la señal isotópica del horizonte más superficial (Andosol 125), se encuentra relacionada en mayor grado a la de la precipitación, en tanto que, los horizontes más profundos reciben menor influencia del agua proveniente de la precipitación de manera directa. En este sentido, el pico de enriquecimiento isotópico que se observa en la muestra de precipitación recolectada el 22 de agosto de 2011 (Figura 5a), se ve reflejado en la señal isotópica del Andosol 1-25 con un desfase de alrededor de dos semanas. Esto sugiere que el agua meteórica que se infiltra en el suelo fluye lateralmente por el horizonte 1 , y la infiltración vertical hacia los horizontes más profundos tiene una menor importancia, aunque se observa una recarga de aguas empobrecidas hacia horizontes más profundos con un desfase de tiempo durante condiciones húmedas. Este hecho puede encontrarse relacionado con la posición del punto de monitoreo sobre la parte media de la ladera donde se encuentra ubicado. 

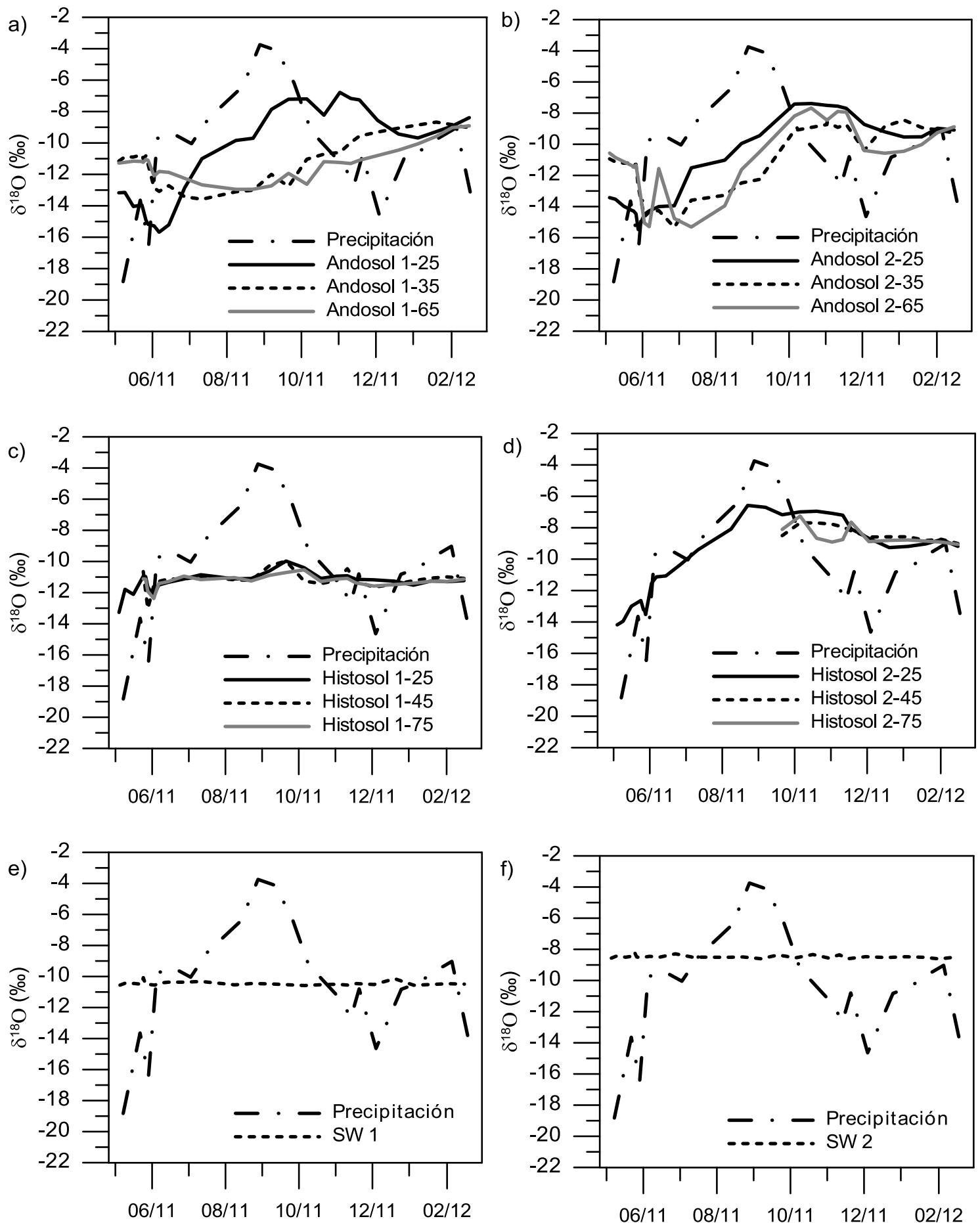

Figura 5. Relación entre las señales isotópicas de $\delta^{18} \mathrm{O}$ de la precipitación y los suelos: a) Andosol 1 , b) Andosol 2, c) Histosol 1, d) Histosol 2, e) Agua de manantial 1 (SW 1), y f) Agua de manantial 2 (SW 2).

Por otro lado, las abundancias isotópicas en los tres horizontes del Andosol 2, guardan relación con la señal de la precipitación, pero con un desfase de tiempo en su respuesta. Esto se puede evidenciar en la Figura 5b, en la cual se observa un desfase de alrededor de 6 semanas entre el pico de enriquecimiento en la abundancia isotópica de $\delta^{18} \mathrm{O}$ en la precipitación y el pico en las muestras tomadas en el Andosol 2 en las tres profundidades monitoreadas. Los valores promedios de las abundancias isotópicas de $\delta^{18} \mathrm{O}$ en los tres niveles del Andosol 2 son muy similares, variando en un rango entre $-11,2 \%$ y $-11,0 \%$ o (Tabla 2). Asimismo, las variaciones máximas entre las señales 
isotópicas de los tres horizontes varían en rangos similares entre ellas, entre $-7,4 \%$ y $-15,5 \%$ en el Andosol 2-25, -8,4\% y -15,5\% en el Andosol 2-35, y -7,7\% y -15,3\%o en el Andosol 2-65, ubicados a 25,35 y $65 \mathrm{~cm}$ de profundidad, respectivamente. Estos valores reflejan que el agua meteórica precipitada se mezcla de mejor manera en los tres horizontes monitoreados en el Andosol 2, lo que se puede atribuir a la ubicación fisiográfica de cada punto de monitoreo de Andosoles, ya que el Andosol 1 se encuentra posicionado en la mitad de la ladera mientras el Andosol 2 se ubica en la parte baja. Este hecho implica que el agua meteórica precipitada tiene mayor posibilidad de infiltrarse verticalmente a medida que la misma va descendiendo hacia la parte baja de la ladera.

En el caso de los Histosoles, en el Histosol 1, las abundancias isotópicas se mantienen mucho más estables en los tres horizontes monitoreados a lo largo del periodo de muestreo, presentando valores promedios de $\delta^{18} \mathrm{O}$ muy similares, con una variación en alrededor de $0,1 \%$ entre ellos. De hecho, los valores promedio están alrededor de -11,3\% para el Histosol 1-25, el Histosol 1-45 y el Histosol 1-75. La señal isotópica de $\delta^{18} \mathrm{O}$ se encuentra mucho más atenuada en el Histosol 1 que en los Andosoles, presentando un rango de variación máximo entre los 3 niveles de alrededor de $3 \%$ a lo largo de todo el periodo monitoreado. Dichos rangos varían entre -10,0\% y -13,3\%o en el Histosol 1-25, -10,3\% y 13,0\% en el Histosol 1-45, y -10,5\% y -12,4\% en el Histosol 1-75. Adicionalmente, la respuesta de las muestras tomadas en el Histosol 1 a la influencia de la señal isotópica de la precipitación es menor, excepto durante épocas lluviosas como durante el evento de lluvia monitoreado a fines de mayo (Figura $5 \mathrm{c}$ ), donde se produce una mezcla rápida que empobrece su señal isotópica de $\delta^{18} \mathrm{O}$ en alrededor de $2 \%$. Estos hechos reflejan que probablemente existe flujo lateral de agua pre-evento que ha estado almacenada por un largo período de tiempo, cuyo origen podría ser un humedal de gran extensión que se encuentra ubicado en la parte alta del punto de muestreo, que recibe agua que proviene de zonas de Andosoles localizadas en las laderas adyacentes.

Por el contrario, el Histosol 2 exhibe una distribución de abundancia isotópica variable, muy relacionada a la señal de la precipitación durante el periodo de muestreo. El valor promedio de $\delta^{18} \mathrm{O}$ del Histosol 2-25 (a $25 \mathrm{~cm}$ de profundidad) es de $-9,9 \%$, con una variación entre $-6,6 \%$ y $-14,2 \%$. Además, las distribuciones de la señal isotópica de $\delta^{18} \mathrm{O}$ son similares en el periodo donde se tienen muestras en los tres horizontes del suelo, lamentablemente no fue posible instalar los platos de succión a 45 y $75 \mathrm{~cm}$ de profundidad antes de septiembre de 2011, una vez que la humedad del terreno fue adecuada para hacerlo (Figura 5d). Los valores promedios de $\delta^{18} \mathrm{O}$ en el Histosol 2-45 y el Histosol 275 (ubicados a $45 \mathrm{~cm}$ y $75 \mathrm{~cm}$ de profundidad) son de $-8,3 \%$ y $-8,6 \%$, respectivamente. Cuyas abundancias isotópicas de $\delta^{18} \mathrm{O}$ varían entre $-7,7 \%$ y $-9,0 \%$ a $45 \mathrm{~cm}$ de profundidad, y -7,3\% y $-9,1 \%$ o a $75 \mathrm{~cm}$ de profundidad. Estos valores de abundancia isotópica en los tres horizontes monitoreados en el Histosol 2 reflejan que debido a que la estación de muestreo se encuentra ubicada a la salida de una zona plana de humedales confinados, dichos horizontes presentan una relación directa con la señal de la precipitación, a diferencia de los tres horizontes monitoreados en el Histosol 1, que al encontrarse ubicado en la parte baja de una zona de ladera cuyo aporte de agua proviene principalmente de zonas de Andosoles adyacentes a ella, no presenta respuesta a la abundancia isotópica de la precipitación. Estos hechos sugieren que el tiempo de residencia en el sistema hidrológico del agua que circula subsuperficialmente por el Histosol 2 es menor al del Histosol 1, es decir, que el agua circula a mayor velocidad por el Histosol 2, principalmente debido a la diferencia en la procedencia del agua que circula por cada estación de monitoreo de Histosoles.

\subsection{Relación entre las señales de $\delta^{18}$ O del agua de lluvia y el agua de manantial}

Los manantiales (Figura 5e y $5 \mathrm{f}$ ) presentan señales isotópicas de $\delta^{18} \mathrm{O}$ muy estables durante todo el periodo de monitoreo, cuyos valores promedio son de $-10,5 \%$ y $-8,5 \%$ para los manantiales 1 (SW1) y 2 (SW2), respectivamente (Tabla 2), con desviaciones estándar de 0,10\% en ambos casos. El proceso de atención generado en su señal isotópica, probablemente deriva de que el agua de los manantiales no presenta reacción a ingresos de precipitación al sistema hidrológico. Lo que sugiere que estos efectivamente se originan desde estratos de roca profundos, y que además, el agua que circula sub-superficialmente por ellos ha estado almacenada por un largo período de tiempo, lo cual supone que poseen altos tiempos de residencia en el sistema hidrológico. También, se puede observar que existe una diferencia de $2 \%$ en los valores promedios de $\delta^{18} \mathrm{O}$ entre ambos manantiales de agua a 
lo largo del periodo de monitoreo (Figuras 5e y 5f, Tabla 2), lo cual sugiere que: i) éstos tienen diferente procedencia, o ii) provienen de diferentes altitudes de recarga.

\subsection{Relación entre las señales de $\delta^{18} \mathrm{O}$ del agua de lluvia y el agua de los ríos}

Las abundancias isotópicas promedio de $\delta^{18} \mathrm{O}$ en los caudales son de $-12,0 \%$ en la M1, $-11,8 \%$ en la M2, - $11,6 \%$ en la M3, - 11,7\%o en la M4, $-10,5 \%$ en la M5, -10,9\%o en la M6, -11,5\%o en la M7, $11,7 \%$ en la M8, $-11,2 \%$ en la M9, y $-11,9 \%$ en la M10. Las variaciones en las señales isotópicas de $\delta^{18} \mathrm{O}$ se encuentran atenuadas en relación a las de la precipitación (Tabla 2) con una variación máxima de 7,7\% en los valores de $\delta^{18} \mathrm{O}$ de la microcuenca 5 durante el periodo de monitoreo.

Basado en estos valores, la ubicación geográfica (Figura 1), y las distribuciones de las señales isotópicas de $\delta^{18} \mathrm{O}$ de las diez microcuencas monitoreadas, se observa que los datos pueden ser reagrupados para su análisis. Las microcuencas M1, M2, M3, M4 y M10 poseen similares distribuciones de señal isotópica durante el periodo de monitoreo. Por lo que pueden ser agrupadas en el subgrupo 1, representado por la microcuenca M10 para el análisis posterior. El subgrupo 1 exhibe variaciones máximas de $1 \%$ para $\delta^{18} \mathrm{O}$, a lo largo de todo el periodo de monitoreo. Las distribuciones isotópicas de las microcuencas M7 y M8 son similares, y pueden reagruparse en el subgrupo 2, el cual será representado por la distribución de señal isotópica de la microcuenca M7. El subgrupo 2 posee variaciones máximas de $0,5 \%$ para $\delta^{18} \mathrm{O}$, y sus valores se encuentran ligeramente enriquecidos en relación a los del subgrupo 1 (aproximadamente $0,8 \%$ ) durante todo el periodo de monitoreo. La distribución de señal isotópica de la microcuenca M6 se encuentra enriquecida en relación a las de los subgrupos 1 y 2 , y ha sido considerada individualmente en el subgrupo 3. De igual forma, la distribución de señal isotópica de la microcuenca M9 difiere de las demás, tendiendo a valores isotópicamente enriquecidos en relación a los de los subgrupos 1 y 2 , y empobrecidos respecto a los del subgrupo 3, por lo que también será analizada individualmente en el subgrupo 4. Finalmente, la distribución de los valores de señal isotópica de la microcuenca M5 muestra una señal isotópica distinta (Figura 6), por lo que será considerada individualmente en el subgrupo 5. Los valores de señal isotópica del subgrupo 5 tienen mayor amplitud respecto a los demás grupos. En general, las señales isotópicas de $\delta^{18} \mathrm{O}$ de los caudales no tienen una relación directa con la señal de la precipitación a lo largo del periodo de monitoreo (Figura 5). Esto, excepto para el subgrupo M5, cuya señal enriquecida en abundancia isotópica, guarda mayor relación con la de la precipitación, incluso manteniendo la misma estacionalidad a lo largo de todo el periodo de monitoreo.

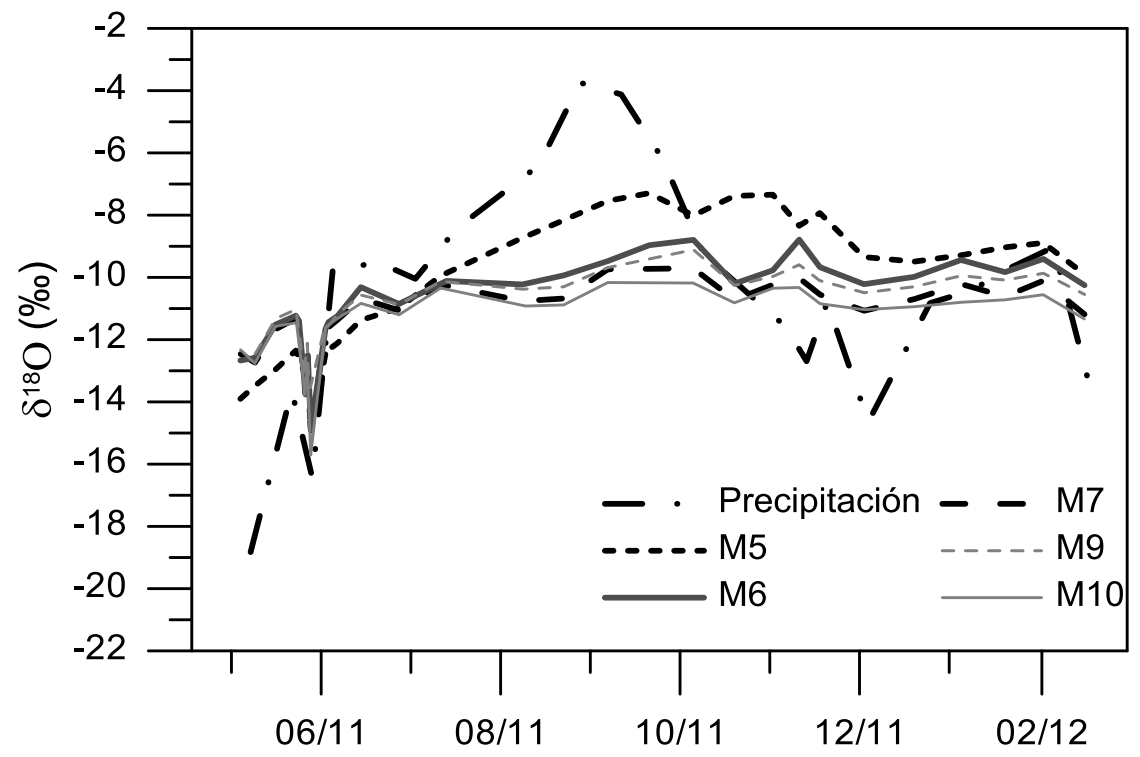

Figura 6. Relación Isotópica de $\delta^{18} \mathrm{O}$ entre la precipitación y los caudales. Subgrupo 1 (M10), Subgrupo 2 (M7), Subgrupo 3 (M6), Subgrupo 4 (M9) y Subgrupo 5 (M5). 
Las señales isotópicas promedio de $\delta^{18} \mathrm{O}$ varían en un rango muy pequeño entre $-11,5 \%$ y $-12,0 \%$ o (Tabla 2) para las microcuencas de la zona oriental de la cuenca de estudio (subgrupos 1 y 2, Figura 6). Esto debido a que las mismas no reciben aporte del agua del Histosol 2 (zona de humedales confinados), que se ubica en la parte alta de la zona oeste del área de estudio. Por otro lado, los valores promedios de $\delta^{18} \mathrm{O}$ en el subgrupo 4 (M9, confluencia de todas las microcuencas), subgrupo 3 (M6) y subgrupo 5 (M5) son de $-11,2 \%$, $-10,9 \%$ y $-10, .5 \%$, respectivamente, los cuales se encuentran ubicados en zonas centro-occidentales del área de estudio. Estos valores reflejan que las abundancias isotópicas de $\delta^{18} \mathrm{O}$ en dichos subgrupos, exhiben un patrón de incremento en sus amplitudes a medida que los puntos de monitoreo se ubican más cerca del Histosol 2.
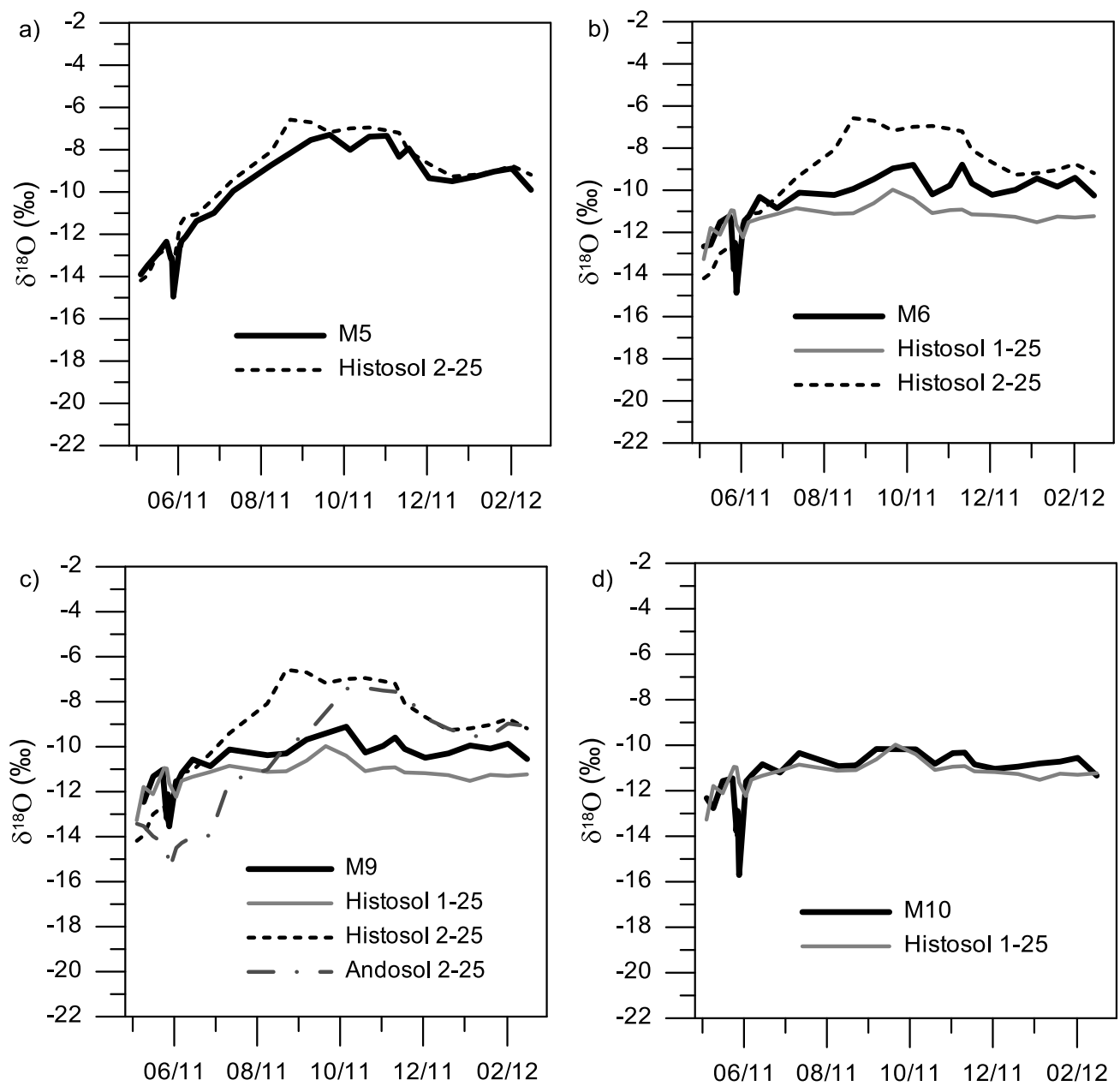

Figura 7. Relación entre las señales isotópicas de $\delta^{18} \mathrm{O}$ de los caudales y suelos; a) M5 vs Histosol 225, b) M6 vs Histosoles 1-25 y 2-25, c) M9 vs Andosol 2-25 e Histosoles 1-25 y 2-25, y d) M10 vs Histosol 1-25.

\subsection{Relación entre las señales de $\delta^{18}$ O del agua del suelo y el agua de los ríos}

En la Figura 7a, se observa que existe una relación directa entre las señales isotópicas de $\delta^{18} \mathrm{O}$ del agua del subgrupo 5 (M5) y la del Histosol 2 (salida de una zona de humedales confinados). Lo que sugiere que dicho Histosol es la principal fuente de aporte de dicho subgrupo durante el periodo de monitoreo. Además, tanto la señal isotópica del agua del Histosol 2 (Figura 5d) como la del subgrupo 5 (Figura 6), responden a la señal del agua de precipitación, sobre todo durante épocas lluviosas. Lo cual muestra que la influencia de la precipitación es mayor en este subgrupo de cuencas que en el resto de microcuencas monitoreadas en el área de estudio.

En las Figuras $7 \mathrm{~b}$ y $7 \mathrm{c}$, se observa que las señales isotópicas de $\delta^{18} \mathrm{O}$ del agua que regula la escorrentía de los subgrupos 3 (M6) y 4 (M9, confluencia de todas las microcuencas en el área de 
estudio), poseen distribuciones intermedias entre la señal isotópica de los Histosoles 1 y 2. Cuyos valores ligeramente más enriquecidos a los del Histosol 1 (entre 0,5\% y 2,5\% para el subgrupo 3 y, $0,5 \%$ y $1,5 \%$ para el subgrupo 4) exhiben la importancia de la mezcla con el agua proveniente del Histosol 2, que posee una mayor abundancia isotópica. En la Figura 7d, se puede notar que a lo largo de todo el periodo de monitoreo, el agua del Histosol 1 tiene una influencia determinante como fuente de aporte a la generación de escorrentía del subgrupo 1 (M10), de igual forma que para las microcuencas del subgrupo 2, que poseen similar tendencia. En contraste, en la Figura 7c, se puede notar que el agua que circula por los Andosoles (representados por el Andosol 1-25) no produce variaciones en la señal isotópica de $\delta^{18} \mathrm{O}$ de $\mathrm{M} 9$. Esto sugiere que el agua que circula por este tipo de suelo no aporta de manera directa a la regulación de la escorrentía en la cuenca, sino más bien, dichos suelos aportan agua a manera de recarga hacia las zonas de los Histosoles en el fondo del valle (Figura 8). Estos hechos reflejan que ambos Histosoles son una importante fuente para la regulación de la escorrentía en la cuenca de estudio, sobretodo durante condiciones normales (periodos menos lluviosos).

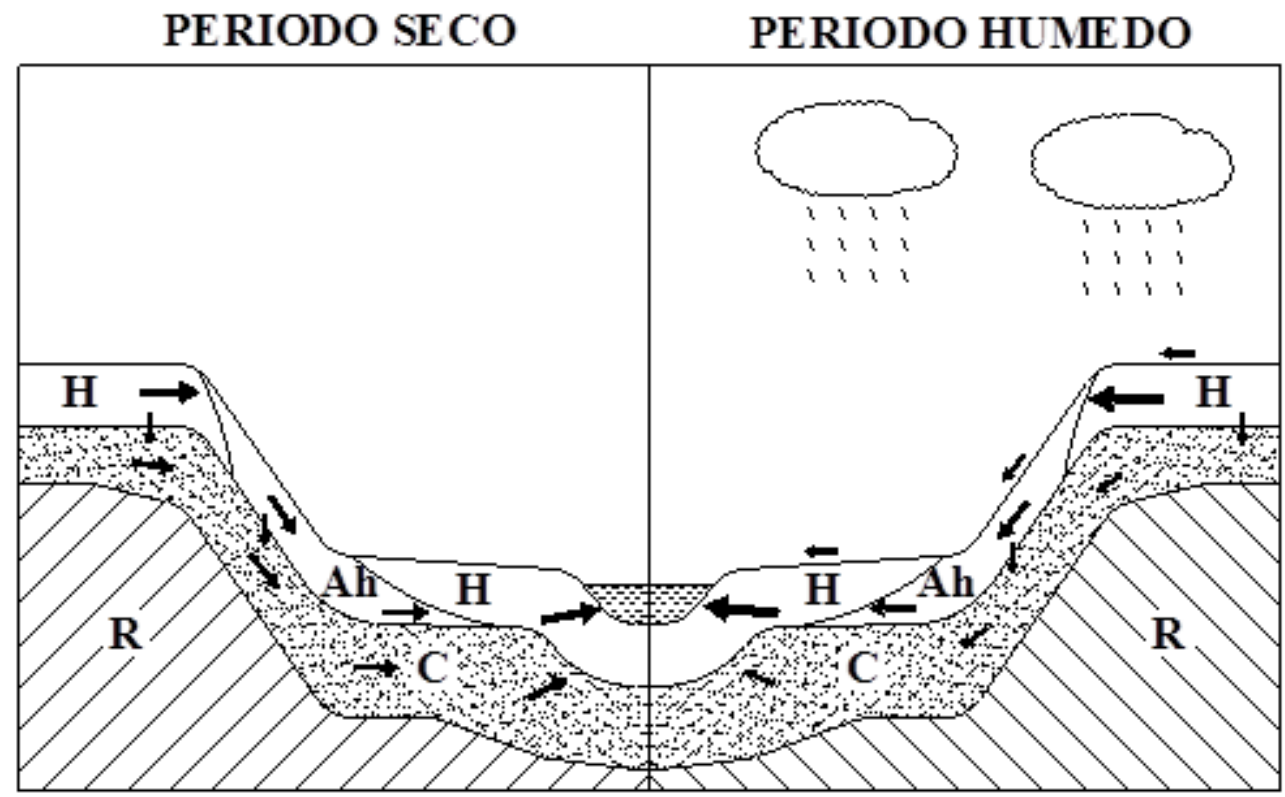

Figura 8. Identificación de las principales fuentes de agua que contribuyen a la generación de escorrentía en la cuenca del Río Zhurucay. (H: Histosol, Ah: Andosol, C: Estrato mineral, R: Estrato rocoso).

\subsection{Identificación de las principales fuentes de escorrentía}

En las Figuras 5 y 6, y, en la Tabla 2 se puede observar que las variaciones de $\delta^{18} \mathrm{O}$ en los caudales y el agua del suelo son menores a las generadas en las muestras de precipitación, efecto que se conoce como atenuación. Dicho efecto sugiere que el agua lluvia no influencia de manera directa a la generación de caudal, sino más bien actúa como fuente de recarga de agua en los suelos; sin embargo, la influencia de la variabilidad temporal de la precipitación sobre la dinámica de los caudales es un proceso que domina la hidrología de los páramos. En consecuencia, la escorrentía superficial por exceso de infiltración es un proceso que no influye a la generación de caudales o se produce de manera localizada durante eventos de lluvia de alta intensidad. Tendencias similares fueron encontradas en los estudios realizados por Crespo y col. (2011a) y Goller y col. (2005) al sur del Ecuador.

Por otra parte, el flujo sub-superficial muestra ser el proceso más influyente en la generación de escorrentía, donde los Histosoles tienen una influencia muy importante en la dinámica hidrológica y ambiental de los páramos del área de estudio. En este sentido, adicionalmente a la dinámica de la lluvia, la humedad antecedente de los Histosoles son determinantes para la generación de caudales pico, donde el flujo superficial por exceso de saturación en las zonas de humedales normalmente 
ocurre bajo condiciones húmedas. De acuerdo a los resultados encontrados en este estudio, el aporte de agua de pre-evento es muy importante en la generación de caudal, aunque su influencia disminuye durante eventos de lluvia de alta intensidad (la Figura 8 muestra un esquema de los principales procesos hidrológicos presentes en condiciones húmedas y secas). Al respecto, Buytaert y Beven (2011) en un estudio realizado en una microcuenca cercana a nuestra zona de estudio, identificaron que el aporte de agua desde zonas de humedales, así como la humedad antecedente del suelo, juegan un papel importante en la rápida dinámica hidrológica de los páramos húmedos del sur del Ecuador (Figura 8) mediante el uso de modelos hidrológicos conceptuales.

De la misma manera, durante condiciones de humedad normal, el agua que fluye a través de los Andosoles recarga los Histosoles localizados en el fondo de los valles (Figura 8). Esta dinámica, en conjunto con las propiedades hidrofísicas de los suelos, son responsables de la regulación del agua que caracteriza a los páramos. Asimismo, tal dinámica condiciona la dinámica ecológica y la calidad del agua, principalmente en la exportación de carbono en el agua. De forma que, aparentemente la hidrología está dominada por un proceso de flujo pistón, donde el agua de evento empuja el agua de eventos previos retenidos en los suelos. Lo anterior está soportado por una mayor exportación de carbono conforme incrementa el caudal (Ochoa y Rodas, 2009).

Por otro lado, durante condiciones secas, el caudal está principalmente controlado por el flujo sub-superficial, donde los Histosoles aparentemente son también aportadores dominantes de agua. Sin embargo, producto de investigaciones anteriores (Crespo y col., 2011a y b), el aporte del agua que fluye por los horizontes minerales y la capa superior alterada de la roca influyen de manera considerable a la generación de caudales. Las aguas provenientes de estratos rocosos más profundos con poca alteración (aguas subterráneas) parecen no contribuir a la generación de caudales en la zona de estudio, aunque de todas formas, estas hipótesis necesitan ser sustentadas de mejor manera.

\section{CONCLUSIONES}

El presente estudio muestra que la aplicación simultánea de múltiples técnicas -como las hidrométricas y las de trazadores- proveen información crucial para la identificación de los principales procesos que gobiernan la dinámica hidrológica de cuencas Andinas. En tal sentido, esta técnica "mixta" ofrece resultados efectivos en la identificación de los mecanismos de generación de escorrentía a escala de microcuenca. Por otro lado, la aplicación del sistema de monitoreo anidado se presenta como un método muy conveniente para la determinación de la variabilidad espacial y temporal de dichos procesos. La combinación de varios métodos reduce la limitación de cada técnica, y al utilizarse de forma conjunta proveen una mayor y mejor comprensión del sistema hidrológico en estudio. Más aún, los trazadores ambientales a diferencia de los datos hidrométricos, permiten una reducción tanto en el período de monitoreo como en los costos de recolección de información. Los trazadores de esta manera ofrecen un prometedor potencial para el análisis de cuencas con poco o sin monitoreo.

Mediante el análisis de las abundancias isotópicas de deuterio y oxígeno-18 en la cuenca de páramo Andino del Río Zhurucay se ha determinado que el fraccionamiento isotópico en la misma se realiza prácticamente en equilibrio, es decir, no existe fraccionamiento por evaporación, y que la precipitación en la cuenca se encuentra influenciada por la reevaporación de lluvias localizadas en la zona de estudio y/o por lluvias provenientes de otras fuentes de vapor de agua, como el océano Pacífico predominantemente. La señal isotópica de la precipitación refleja la existencia de los efectos: i) altitudinal, empobrecimiento en abundancia isotópica al aumentar la altitud, ii) de cantidad, relacionado al empobrecimiento en la señal isotópica de la precipitación debido al ingreso localizado de altas cantidades de lluvia en algunas épocas en las diferentes partes de la cuenca, y iii) de estacionalidad, evidenciado por el empobrecimiento en abundancia isotópica durante épocas lluviosas, y el enriquecimiento en la misma durante periodos secos.

En relación a la identificación de las fuentes de agua que contribuyen a la generación de escorrentía, los resultados revelan que el agua de pre-evento es la principal fuente de generación de escorrentía durante épocas lluviosas, siendo los Histosoles las principales fuentes de aporte a la descarga. Los Andosoles tienen como función principal la de regular el caudal y recargar los 
Histosoles durante condiciones normales, principalmente debido a su posición en el paisaje (sobre los Histosoles). Por otra parte, durante condiciones de menor humedad los resultados sugieren que los Histosoles son la principal fuente de aporte a la generación de caudal. De la misma forma, no existen evidencias de aporte significativo de agua subterránea a la generación de caudal en la zona, básicamente debido al tipo de geología encontrado.

\section{AGRADECIMIENTOS}

Esta investigación fue posible gracias al financiamiento de la DIUC (Dirección de Investigación de la Universidad de Cuenca) proyecto "Estudio bio-hidrológico de un ecosistema de páramo húmedo Andino", la SENESCYT (Secretaría Nacional de Educación Superior, Ciencia, Tecnología e Innovación) proyecto PIC-11-715 y la DFG (German Research Foundation, Research Unit 816: Biodiversity and Sustainable Management of a Megadiverse Mountain Ecosystem in South Ecuador, subproject B3.2, BR2238/4-1). Los autores agradecen la colaboración de IAMGOLD Ecuador S.A. (Proyecto Quimsacocha) en la logística de la investigación, y los valiosos comentarios del Profesor Jan Feyen, que ayudaron a mejorar el manuscrito.

\section{BIBLIOGRAFÍA}

Baculima, F.L., J.C. Baculima, W.A. Bermeo, 1999. Caracterización de clima por microcuencas en el Austro Ecuatoriano. Tesis de Ingeniería sin publicar. Facultad de Ingeniería, Universidad de Cuenca, Cuenca, Ecuador.

Blume, T., E. Zehe, A. Bronstert, 2007. Rainfall runoff response, event-based runoff coefficients and hydrograph separation. Hydrol. Sci. J., 52(5), 843-862.

Buytaert, W., 2004a. The properties of the soils of the south Ecuadorian páramo and the impact of land use changes on their hydrology. PhD thesis, Faculty of Agricultural and Applied Biological Sciences, Katholieke Universiteit Leuven, Leuven, Belgium.

Buytaert, W., B. De Bièvre, G. Wyseure, J. Deckers, 2004b. The use of the linear reservoir concept to quantify the impact of land use changes on the hydrology of catchments in the Ecuadorian Andes. Hydrol. Earth Syst. Sc., 8, 108-114.

Buytaert, W., J. Sevink, B.D. Leeuw, J. Deckers, 2005. Clay mineralogy of the soils in the south Ecuadorian paramo region. Geoderma, 127, 114-129.

Buytaert, W., R. Célleri, B. De Bièvre, F. Cisneros, 2006a. Hidrología del páramo andino: propiedades, importancia y vulnerabilidad. Descargado de ftp://ftp.ciat.cgiar.org/.../ HIDROLOGIA_DEL_PARAMO en octubre 2010, 29 pp.

Buytaert, W., R. Célleri, B. De Bièvre, F. Cisneros, G. Wyseure, J. Deckers, R. Hofstede, 2006b. Human impact on the hydrology of the Andean páramos. Earth-Sci. Rev., 79, 53-72.

Buytaert, W., V. Iniguez, B. De Bièvre, 2007. The effects of afforestation and cultivation on water yield in the Andean páramo. Forest Ecol. Manag., 251, 22-30.

Buytaert, W., K. Beven, 2011. Models as multiple working hypotheses: Hydrological simulation of tropical alpine wetlands. Hydrol. Process., 25(11), 1784-1799.

Castaño, C., 2002. Páramos y ecosistemas alto andinos de Colombia en condición hotspot \& global climatic tensor. Ministerio del Medio Ambiente and Instituto de Hidrología, Meteorología y Estudios Ambientales, Bogotá, Colombia, 387 pp.

Crespo P., R. Célleri, W. Buytaert, J. Feyen, V. Iñiguez, P. Borja y B. de Bièvre, 2010. Land use change impacts on the hydrology of wet Andean páramo ecosystems. IAHS Publ. 336. 
Crespo, P., A. Bücker, J. Feyen, K.B. Vaché, H-G., Frede, L. Breuer, 2011a. Preliminary evaluation of the runoff processes in a remote montane cloud forest basin using Mixing Model Analysis and Mean Transit Time. Hydrol. Process., 26(25), 3896-3910.

Crespo, P., J. Feyen, W. Buytaert, A. Bücker, L. Breuer, H-G. Frede, M. Ramírez, 2011b. Identifying controls of the hydrological response of small catchments in the tropical Andes (Ecuador). $J$. Hydrol., 407, 164-174.

De Bièvre, B., V. Iñiguez, W. Buytaert, 2006. Hidrología del páramo. Importancia, propiedades y vulnerabilidad. Órgano de Difusión del Grupo de Trabajo en Páramos del Ecuador (GTP), Investigaciones biofísicas de páramo, 21, 71 pp. Descargado de http://www.ecociencia.org/.../paramo-GTP21-091128.pdf en abril 2007.

Díaz-Granados Ortiz, M.A., J.D. Navarrete Gonzalez, T. Suárez López, 2005. Páramos: Sensitive hydrosystems. Universidad de los Andes, Facultad de Ingeniería, Revista de Ingeniería, 22, 6475.

FAO/ISRIC/ISSS, 1998. World Reference Base for Soil Resources. World Soil Resources Reports, FAO (84), 88.

García, M., F. Villalba, L. Araguás-Araguás, K. Rozanski, 1998. The role of atmospheric circulation patterns in controlling the regional distribution of stable isotope contents in precipitation. Preliminary results from two transects in the Ecuadorian Andes. Isotope Techniques in the Study of Environmental Change, Proc. Series, International Atomic Energy Agency, Vienna, IAEASM-349/7, 127-140.

Goller, R., W. Wilcke, M.J. Leng, H.J. Tobschall, K. Wagner, C. Valarezo, W. Zech, 2005. Tracing water paths through small catchments under a tropical montane rain forest in south Ecuador by an oxygen isotope approach. J. Hydrol., 308, 67-80.

Hofstede, R.G., 1995. Effects or Burning and Grazing on a Colombian Paramo Ecosystem. PhD Thesis, Universitet van Amsterdam, Amsterdam, The Netherlands, 198 pp.

Kendal, C., J. McDonnell (Eds.), 1998. Isotope tracers in catchment hydrology. Elsevier Science B.V., Amsterdam, The Netherlands, 839 pp.

Medina, G., V.P. Mena, 2001. Los páramos en el Ecuador. En: Mena V.P., G. Medina, R. Hofstede (Eds.). Los páramos del Ecuador particularidades, problemas y perspectivas. Abya Yala/Proyecto Páramos, Quito, Ecuador, 20 pp.

Mena, P., R. Hofstede, 2006. Los páramos ecuatorianos. En: Moraes, M. R., B. Øllgaard, L.P. Kvist, F. Borchsenius, H. Balslev (Eds.), Botánica Económica de los Andes Centrales. Universidad Mayor de San Andrés, La Paz, Bolivia, 91-109.

Ochoa, A., V. Rodas, 2009. Variabilidad espacio-temporal de la calidad del agua en cuatro microcuencas de los páramos de Quimsacocha. Tesis BSc sin publicar. Facultad de Ingeniería, Universidad de Cuenca, Cuenca, Ecuador, 107 pp.

PROMAS/IAMGOLD, 2009. Elaboración de la línea base en hidrología de los páramos de Quimsacocha y su área de influencia. Tech. Rep., PROMAS, Universidad de Cuenca, Cuenca, Ecuador, 68 pp.

Quichimbo, P., I. Cárdenas, G. Tenorio, P. Crespo, P. Borja, R. Célleri, 2011. Efectos del cambio de cobertura vegetal sobre las propiedades hidrofísicas del suelo en un área de páramo, sur del Ecuador. Programa CYTED, Buenos Aires, Argentina, 255-265.

Roche, M., R. Gonfiantini, J. Fontes, N. Abasto, L. Noriega, 1999. The isotopic composition of precipitation on the Andes and Amazon of Bolivia. Isotope Techniques in Water Resources Development and Management, Organismo Internacional de Energía Atómica, Vienna, Austria.

Rozanski, K., L. Araguás-Araguás, 1995. Spatial and temporal variability of stable isotope composition of precipitation over the south american continent. Bull. Inst. Fr. d'Études Andines, 24, 379-390.

Van der Hammen, T., H. Hooghiemstra, 2000. Neogene and quaternary history of vegetation, climate and plant diversity in Amazonia. Quaternary Sci. Rev., 19, 725-742. 
Vogel, J., J. Lerman, W. Mook, 1975. Natural isotopes in surface and groundwater from Argentina. Hydrol. Sci. Bull. , 20, 203-221.

Vuille, M., R.S. Bradley, F. Keimig, 2000. Interannual climate variability in the Central Andes and its relation to tropical Pacific and Atlantic forcing. J. Geophys. Res-Atmos., 105(D10), 12447-12460

Wilcox, B., 2011. La investigación Hidrológica en los altos andes: La ruta por delante. Descargado de http://www.condesan.org/e-foros/paramos2/lainvestigacionhidrologicaMay24.htm en agosto 2012.

Yurtsever, Y. (Ed. Coord.), 2000. Prefacio de la Sección VI: Modelación. En: Mook, W.G. (Ed.) Isotopos ambientales en el ciclo hidrológico: Principios y aplicaciones. Sección de Hidrología Isotópica, Organismo Internacional de Energía Atómica, Viena, Austria, 1 pp. 